PROCEEDINGS OF THE AMERICAN MATHEMATICAL SOCIETY

Volume 124, Number 1, January 1996

\title{
ON THE SOLVABILITY OF A NONLINEAR SECOND-ORDER ELLIPTIC EQUATION AT RESONANCE
}

\author{
CHUNG-CHENG KUO
}

(Communicated by Jeffrey Rauch)

\begin{abstract}
We study the existence of solutions of the Neumann problem for semilinear second-order elliptic equations at resonance in which the nonlinear terms may grow superlinearly in one of the directions $u \rightarrow \infty$ and $u \rightarrow-\infty$, and sublinearly in the other. Solvability results are obtained under assumptions either with or without a Landesman-Lazer condition. The proofs are based on degree-theoretic arguments.
\end{abstract}

\section{INTRODUCTION}

Let $\Omega \subset \mathbf{R}^{N}(N \geq 2)$ be a smooth bounded domain in $\mathbf{R}^{N}$. In this paper we consider the Neumann problem

$$
\Delta u+g(x, u)=h(x) \quad \text { in } \Omega, \quad \frac{\partial u}{\partial n}=0 \quad \text { on } \partial \Omega
$$

where $\Delta$ denotes the Laplacian on $\mathbf{R}^{N}(N \geq 2), h \in L^{p}(\Omega)(p>N / 2)$ is given, $\frac{\partial}{\partial n}$ denotes the outward normal derivative on $\partial \Omega$ and $g: \Omega \times \mathbf{R} \rightarrow \mathbf{R}$ is a Caratheodory function, that is, $g(x, u)$ is continuous in $u \in \mathbf{R}$ for almost all $x \in \Omega$, is measurable in $x \in \Omega$ for all $u \in \mathbf{R}$ and satisfies for each $r>0$ there exists $a_{r} \in L^{p}(\Omega)$ such that

$$
|g(x, u)| \leq a_{r}(x)
$$

for a.e. $x \in \Omega$ and all $|u| \leq r$. The solvability of the problem (1.1) has been extensively studied when the nonlinearity $g$ is assumed to have linear growth in $u$ (see $[2,3,4,5]$ ). When $g$ is allowed to grow superlinearly in $u$ in one of the directions $u \rightarrow \infty$ and $u \rightarrow-\infty$, and bounded in $L^{p}(\Omega)$ in the other, existence theorems for a solution to (1.1) are proved in $[6,9]$ under assumptions with a Landesman-Lazer condition (see (2.1) below) originated in [7]. The purpose of this paper is to give a solvability condition for (1.1)when $g$ is allowed to grow superlinearly in $u$ in one of the directions $u \rightarrow \infty$ and $u \rightarrow-\infty$, and sublinearly in the other. More precisely, we assume that $p>N / 2$ and

$(\mathbf{H})$ There exist constants $a, \alpha, \beta, r_{0} \geq 0, \beta<1,(\alpha(p-1)+\beta) / p \leq 1$, and $b, c, d \in L^{p}(\Omega), b \geq 0$, such that for a.e. $x \in \Omega, u \geq r_{0}$

$$
c(x) \leq g(x, u) \leq a|u|^{\alpha}+b(x),
$$

Received by the editors June 8, 1994

1991 Mathematics Subject Classification. Primary 35J65, 47H11, 47H15.

Key words and phrases. Second-order elliptic equation, Landesman-Lazer condition, LeraySchauder degree.

This research was supported in part by the National Science Council of the Republic of China.

(C)1996 American Mathematical Society 
and for a.e. $x \in \Omega, u \leq-r_{0}$

$$
-a|u|^{\beta}-b(x) \leq g(x, u) \leq d(x) .
$$

Based on degree-theoretic arguments (see [8]), we obtain solvability results under assumptions either with or without a Landesman-Lazer condition. The results which remain valid when $g$ is replaced by $-g$ in $(\mathbf{H})$ complement those cited above.

In what follows we shall make use of the real Banach spaces $L^{p}(\Omega), C(\bar{\Omega})$ with the norms denoted by $\|u\|_{\nu},\|u\|_{c}$, respectively, and the Sobolev space $W^{2, p}(\Omega)$. The continuous imbedding $C(\bar{\Omega}) \rightarrow L^{p}(\Omega)$ for $p \geq 1$ and the compact imbedding $W^{2, p}(\Omega) \rightarrow C(\bar{\Omega})$ for $p>N / 2$ have been noted below. By a solution of (1.1), we mean a function $u \in W^{2, p}(\Omega)$ satisfies the differential equation in (1.1) a.e. on $\Omega$. For a linear operator $L$, we denote by $N(L)$ and $R(L)$ the null space and the range of $L$, respectively. From the Fredholm alternative theorem we have $N^{\perp}(\Delta)=R(\Delta)$ and $R^{\perp}(\Delta)=N(\Delta)=\langle 1\rangle$. Finally, the following result is well known (cf. [1]):

For each $p>1$, there exists $C_{p}>0$ such that

$$
\|u-P u\|_{W^{2, p}(\Omega)} \leq C_{p}\|\Delta u\|_{L^{p}}
$$

for all $u \in W^{2,2}(\Omega), \frac{\partial u}{\partial n}=0$ on $\partial \Omega$ with $\Delta \in L^{p}(\Omega)$, where $P: L^{2}(\Omega) \rightarrow N(\Delta)$, $P u=\int_{\Omega} u /|\Omega|$ for $u \in L^{2}(\Omega)$.

\section{EXISTENCE RESUlts}

The main result is the following Theorem 1 which is an existence theorem for a solution of (1.1) satisfying the Landesman-Lazer condition. By modifying slightly the proof of Theorem 1 and the solvability conditions of (1.1), we obtain Theorems 2 and 4 and also Corollary 3 when the Landesman-Lazer condition is excluded.

Theorem 1. Let $p>N / 2$ and $g: \Omega \times \mathbf{R} \rightarrow \mathbf{R}$ be a Caratheodory function satisfying $(\mathbf{H})$. Then the problem (1.1) has a solution $u \in W^{2, p}(\Omega)$ for any $h \in L^{p}(\Omega)$ provided that

$$
\int g_{-}<\int h<\int g_{+}
$$

where $g_{+}(x)=\lim _{u \rightarrow \infty} \inf g(x, u), g_{-}(x)=\lim _{u \rightarrow-\infty} \sup g(x, u)$.

Proof. We assume that the Lebesgue measure $|\Omega|=1$ and let $f: \mathbf{R} \rightarrow \mathbf{R}$ be a continuous function defined by

$$
f(u)= \begin{cases}u & \text { if }|u| \leq 1, \\ \frac{u}{|u|} & \text { if }|u|>1 .\end{cases}
$$

We consider the boundary value problems

$$
\Delta u+(1-t) f\left(\int u\right)+\operatorname{tg}(x, u)=\operatorname{th}(x) \quad \text { in } \Omega, \quad \frac{\partial u}{\partial n}=0 \quad \text { on } \partial \Omega
$$

for $0 \leq t \leq 1$. The problem (2.2) has only a trivial solution when $t=0$, and becomes the original problem (1.1) when $t=1$. To apply the Leray-Schauder degree theory, it requires an a priori bound. If we can show that there exists $R_{0}>0$ such that $\|u\|_{c}<R_{0}$ for all possible solutions $u$ to (2.2) for all $0<t<1$, then using degreetheoretic arguments it follows that the problem (1.1) has a solution $u$ such that $\|u\|_{c}<R_{0}$. That would prove the theorem. 
We note first that there exists Caratheodory functions $g_{1}, g_{2}: \Omega \times \mathbf{R} \rightarrow \mathbf{R}$ such that

$$
\begin{gathered}
g=g_{1}+g_{2} \quad \text { and } \quad 0 \leq g_{1}(x, u) \leq a|u|^{\alpha}, \\
\left|g_{2}(x, u)\right| \leq a|u|^{\beta}+e(x), \quad e(x)=\max \left\{a_{r_{0}}, b(x),|c(x)|,|d(x)|\right\}
\end{gathered}
$$

for almost all $x \in \Omega$ and all $u \in \mathbf{R}$. This may be done by defining

$$
g_{1}(x, u)=\min \left\{g(x, u)+a|u|^{\beta}+e(x), a|u|^{\alpha}\right\} \theta(u) \text { and } g_{2}=g-g_{1},
$$

where $\theta: \mathbf{R} \rightarrow \mathbf{R}$ is a continuous function such that for $u \in \mathbf{R} 0 \leq \theta(u) \leq 1$, $\theta(u)=0$ for $|u| \leqq r_{0}$ and $\theta(u)=1$ for $|u| \geqq 2 r_{0}$.

Let $u$ be a possible solution to $(2.2)$ for some $0<t<1$. Then $u$ satisfies $(2.3)$ for $x \in \Omega$. By integrating (2.2) over $\Omega$, we have

$$
(1-t) f\left(\int u\right)+t \int_{\Omega} g(x, u(x)) d x=t \int_{\Omega} h .
$$

Thus by (2.3) we have

$$
t \int g_{1}(x, u) \leq \int\left|g_{2}(x, u)\right|+1+\int|h| \leq C_{2}\|u\|_{C}^{\beta}+C_{3}
$$

and

$$
\begin{aligned}
t^{p} \int\left|g_{1}(x, u)\right|^{p} & \leq t \int g_{1}(x, u)(a|u|)^{\alpha(p-1)} \\
& \leq\left(a\|u\|_{c}\right)^{\alpha(p-1)}\left(C_{2}\|u\|_{C}^{\beta}+C_{3}\right) \\
& \leq C_{4}\left(\|u\|_{C}^{\alpha(p-1)}+\|u\|_{C}^{\alpha(p-1)+\beta}\right)
\end{aligned}
$$

for some constants $C_{2}, C_{3}, C_{4}$ independent of $u$. It follows from (2.4), (2.5) that

$$
t\left\|g_{1}(x, u)\right\|_{L^{p}} \leq C_{5}\left(\|u\|_{C}^{(\alpha(p-1)) / p}+\|u\|_{C}^{(\alpha(p-1)+\beta) / p}\right)
$$

for some constant $C_{5}>0$ independent of $u$. Hence there exists a constant $C_{6}>0$ such that

$$
\begin{aligned}
\|\Delta u\|_{L^{p}} & =\left\|\operatorname{th}-\operatorname{tg}(x, u)-(1-t) f\left(\int u\right)\right\|_{L^{p}} \\
& \leq C_{6}\left(1+\|u\|_{C}^{\alpha(p-1) / p}+\|u\|_{C}^{(\alpha(p-1)+\beta) / p}+\|u\|_{C}^{\beta}\right)
\end{aligned}
$$

holds for all possible solutions $u$ of (2.2) for some $0 \leq t \leq 1$. Finally we shall show that the solutions of (2.2) for all $0<t<1$ have an a priori bound in $C(\bar{\Omega})$. If this is not true, then there exists a sequence $\left\{u_{n}\right\}$ in $W^{2, p}(\Omega)$ and a sequence $\left\{t_{n}\right\}$ in $(0,1)$ such that $u_{n}$ satisfies $(2.2)$ with $t=t_{n}$ and $\left\|u_{n}\right\|_{c} \geq n$ for all $n$. Let $v_{n}=u_{n} /\left\|u_{n}\right\|_{C}$. Then $\left\|v_{n}\right\|_{C}=1$ and by (2.8) we have

$$
\left\|\Delta v_{n}\right\|_{L^{P}} \leq C_{6}\left(1+\left\|u_{n}\right\|_{C}^{\alpha(p-1) / p}+\left\|u_{n}\right\|_{C}^{(\alpha(p-1)+\beta) / p}+\left\|u_{n}\right\|_{C}^{\beta}\right) /\left\|u_{n}\right\|_{C} .
$$

With the hypothesis $(\alpha(p-1)+\beta) / p \leqq 1$ and $\beta<1$, the right-hand side of $(2.9)$ is bounded by a constant independent of $n$ and hence by $(1.2),(I-P) v_{n}$ has a subsequence convergent in $C(\bar{\Omega})$. Because $\left\{\int v_{n}\right\}$ is bounded, we may assume without loss of generality that $\left\{v_{n}\right\}$ converges to $w$ in $C(\bar{\Omega})$ for some $w \in C(\bar{\Omega})$ such that $w \neq 0$ because of $\left\|v_{n}\right\|_{C}=1$. By $(2.7)$ the sequence $g_{1}\left(x, u_{n}(x)\right) /\left\|u_{n}\right\|_{C}$ has a subsequence convergent weakly in $L^{p}(\Omega)$, and

$$
\left(t_{n} h-t_{n} g_{2}\left(x, u_{n}(x)\right)-\left(1-t_{n}\right) f\left(\int u_{n}\right)\right) /\left\|u_{n}\right\|_{C} \rightarrow 0 \quad \text { as } n \rightarrow \infty
$$


thus we may assume that $m \in L^{p}(\Omega)$,

$$
\left[-t_{n} h+t_{n} g\left(x, u_{n}(x)\right)+\left(1-t_{n}\right) f\left(\int u_{n}\right)\right] /\left\|u_{n}\right\|_{C} \rightarrow m(x)
$$

weakly in $L^{p}(\Omega)$. Since $\Delta$ is also weakly closed, it follows that $w \in W^{2, p}(\Omega)$ and

$$
\Delta w+m=0 \quad \text { in } \Omega,\left.\quad \frac{\partial w}{\partial n}\right|_{\partial \Omega}=0 .
$$

We deduce using (2.3) that $m(x) \geq 0$ for a.e. $x \in \Omega$. By $(2.10)$ we have $\int m=0$ so that $m(x)=0$ for a.e. $x \in \Omega$. Consequently, $w \equiv w_{0}$ for some constant $w_{0} \neq 0$. We consider only the case $w_{0}>0$, for the case $w_{0}<0$ can be treated similarly. Therefore, by the properties of $N(\Delta)$ there exists an $n_{0} \in \mathbf{N}$ such that $v_{n}>w_{0} / 2$ in $\bar{\Omega}$ for all $n \geq n_{0}$, and hence $u_{n}(x) \rightarrow \infty$ for each $x \in \Omega$. Integrating (2.2) when $u=u_{n}$ and $t=t_{n}$ we have

$$
t_{n} \int g\left(x, u_{n}\right)<\left(1-t_{n}\right) f\left(\int u_{n}\right)+t_{n} \int g\left(x, u_{n}\right)=t_{n} \int h,
$$

since $t_{n} \neq 0$, and by $(\mathbf{H})$ that $g\left(x, u_{n}\right)$ is bounded from below by a $L^{1}(\Omega)$-function independent of $n$ for $n$ large enough. Applying the Fatou lemma to the inequality

$$
\int g\left(x, u_{n}\right)<\int h
$$

we have

$$
\int g_{+} \leq \int h
$$

which contradicts the second inequality in (2.1). This completes the proof of Theorem 1.

An interesting case in which (2.1) is not satisfied is when

$$
\left\{\begin{array} { l } 
{ c ( x ) = g _ { + } ( x ) \quad \text { for a.e. } x \in \Omega , } \\
{ \int h = \int c }
\end{array} \quad \text { or } \quad \left\{\begin{array}{l}
d(x)=g_{-}(x) \quad \text { a.e. } x \in \Omega, \\
\int h=\int d .
\end{array}\right.\right.
$$

By slightly modifying the proof of Theorem 1, we obtain the following theorem and corollary.

Theorem 2. Let $p>N / 2$ and $g: \Omega \times \mathbf{R} \rightarrow \mathbf{R}$ be a Caratheodory function satisfying $(\mathbf{H})$. Then the problem (1.1) has a solution $u \in W^{2, P}(\Omega)$ for any $h \in L^{p}(\Omega)$ such that

$$
\int d \leq \int h \leq \int c .
$$

Proof. In proving Theorem 1, the condition (2.1) is used only in the final part of the proof to produce contradictions. Thus we can proceed in exactly the same way as the proof of Theorem 1, up to the point where we choose the case $w_{0}>0$ to consider and obtain results $(2.11)$ and $u_{n}>r_{0}$ in $\bar{\Omega}$ for $n$ large enough. It follows that

$$
\int c \leq \int g\left(x, u_{n}\right)<\int h,
$$

which contradicts (2.12). This completes the proof of the theorem. 
Corollary 3. Let $p>N / 2$ and $g: \Omega \times \mathbf{R} \rightarrow \mathbf{R}$ be a Caratheodory function satisfying $(\mathbf{H})$. If for almost all $x \in \Omega, u \in \mathbf{R},|u| \geqq r_{0}, u g(x, u) \geq 0$ holds, then the problem (1.1) has a solution $u \in W^{2, p}(\Omega)$ for any $h \in L^{p}(\Omega)$ such that $\int h=0$.

Finally, we combine (2.1) with (2.12) to produce new solvability conditions. The following theorem can be obtained.

Theorem 4. Let $p>N / 2$ and $g: \Omega \times \mathbf{R} \rightarrow \mathbf{R}$ be a Caratheodory function satisfying $(\mathbf{H})$. Then the problem (1.1) has a solution $u \in W^{2, P}(\Omega)$ for any $h \in L^{p}(\Omega)$ such that either $\int g_{-}<\int h \leq \int c$ or $\int d \leq \int h<\int g_{+}$holds.

Remarks. 1. Theorem 1 and Corollary 3 are obvious improvements of $[2,4,6,9]$ and $[3,5]$, respectively.

2. By carefully examining our proof, it can be shown that our results are still true if $\frac{\alpha(p-1)+\beta}{p}<1$ in $(\mathbf{H})$ and $p>N / 2$ is replaced by $P \geq N / 2(N \geq 3)$.

\section{REFERENCES}

1. S. Agmon, A. Douglis, and L. Nirenberg, Estimates near the boundary for solutions of elliptic partial differential equations satisfying general boundary conditions I, Comm. Pure Appl. Math. 12 (1959), 623-727. MR 23:A2610

2. H. Berestycki and D. G. de Figueiredo, Double resonance in semilinear elliptic equations, Comm. Partial Differential Equations. 6 (1981), 91-22. MR 82f:35078

3. C. P. Gupta, Perturbation of second order elliptic problems by unbounded nonlinearities, Nonlinear Anal. 6 (1982), 919-933. MR 84c:35042

4. N. Hirano, Nonlinear perturbations of second order linear elliptic boundary value problems, Houston J. Math. 14 (1988), 105-114. MR 90c:35091

5. R. Iannacci, M. N. Nkashama, and J. R. Ward, Jr., Nonlinear second order elliptic partial differential equations at resonance, Trans. Amer. Math. Soc. 311 (1989), 711-726. MR 90a:35091

6. R. Kannan and R. Ortega, Landesman-Lazer conditions for problems with "one-side unbounded" nonlinearities, Nonlinear Anal.9 (1985), 1313-1317. MR 87d:35051

7. E. M. Landesman and A. C. Lazer, Nonlinear perturbations of linear elliptic boundary value problems at resonance, J. Math. Mech. 19 (1970), 609-623. MR 42:2171

8. N. G. Lloyd, Degree theory, Cambridge Univ. Press, Cambridge, 1978. MR 58:12558

9. J. R. Ward, Jr., Perturbations with some superlinear growth for a class of second order elliptic boundary value problems, Nonlinear Anal. 6 (1982), 367-374. MR 83i:35076

Department of Mathematics, Fu Jen University, Taipei, Taiwan 